\title{
Correction to: YTHDF2 reduction fuels inflammation and vascular abnormalization in hepatocellular carcinoma
}

Jiajie Hou ${ }^{1,2,3,4^{*+}}$, He Zhang ${ }^{1,5+}$, Jun Liu ${ }^{6+}$, Zhenjun Zhao ${ }^{1}$, Jianye Wang ${ }^{1}$, Zhike Lu' ${ }^{6}$, Bian Hu' ${ }^{7}$, Jiankui Zhou ${ }^{7}$, Zhicong Zhao ${ }^{1}$, Mingxuan Feng ${ }^{1}$, Haiyan Zhang ${ }^{4,8}$, Bin Shen ${ }^{9}$, Xingxu Huang ${ }^{7}$, Beicheng Sun ${ }^{2}$, Mark J. Smyth ${ }^{8}$, Chuan $\mathrm{He}^{6,10^{*}}$ and Qiang Xia ${ }^{1 *}$

\section{Correction to: Mol Cancer 18, 163 (2019) https://doi.org/10.1186/s12943-019-1082-3}

Following the publication of the original article [1], the author list lacked a co-author's name: Mark J. Smyth, and his affiliation is QIMR Berghofer Medical Research Institute. The correct author list should be "Jiajie Hou; He Zhang; Jun Liu; Zhenjun Zhao; Jianye Wang; Zhike Lu; Bian Hu; Jiankui Zhou; Zhicong Zhao; Mingxuan Feng; Haiyan Zhang; Bin Shen; Xingxu Huang; Beicheng Sun; Mark J. Smyth; Chuan He; Qiang Xia". The error has been corrected in the HTML and PDF version of this article.
China. ${ }^{8}$ Immunology of Cancer and Infection Laboratory, QIMR Berghofer Medical Research Institute, Herston, Queensland 4006, Australia. ${ }^{9}$ Key Laboratory of Reproductive Medicine, Department of Histology and Embryology, Nanjing Medical University, Nanjing 211166, China. ${ }^{10}$ Howard Hughes Medical Institute, University of Chicago, Chicago, IL 60637, USA.

Published online: 04 September 2020

\section{Reference}

1. Hou J, Zhang H, Liu J, et al. YTHDF2 reduction fuels inflammation and vascular abnormalization in hepatocellular carcinoma. Mol Cancer. 2019;18: 163 https://doi.org/10.1186/s12943-019-1082-3.

\footnotetext{
Author details

'Department of Liver Surgery, Renji Hospital, School of Medicine, Shanghai Jiaotong University, Shanghai 200127, China. ${ }^{2}$ Department of Hepatobiliary Surgery, The Affiliated Drum Tower Hospital of Nanjing University Medical School, Nanjing 210093, China. ${ }^{3}$ Department of Hepatobiliary Surgery, Sun Yat-sen University Cancer Center, Guangzhou 510060, China. ${ }^{4}$ State Key Laboratory of Oncology in South China, Sun Yat-sen University Cancer Center, Guangzhou 510060, China. ${ }^{5}$ Department of Surgery, The University of Hong Kong-Shenzhen Hospital, Shenzhen 518053, China. ${ }^{6}$ Department of Chemistry, Department of Biochemistry and Molecular Biology, Institute for Biophysical Dynamics, University of Chicago, Chicago, IL 60637, USA. ${ }^{7}$ School of Life Science and Technology, ShanghaiTech University, Shanghai 201210,

The original article can be found online at https://doi.org/10.1186/s12943019-1082-3.

*Correspondence: houjj@sysucc.org.cn; chuanhe@uchicago.edu; xiaqiang@shsmu.edu.cn

${ }^{\dagger}$ Jiajie Hou, He Zhang and Jun Liu contributed equally to this work. 'Department of Liver Surgery, Renji Hospital, School of Medicine, Shanghai Jiaotong University, Shanghai 200127, China

${ }^{6}$ Department of Chemistry, Department of Biochemistry and Molecular Biology, Institute for Biophysical Dynamics, University of Chicago, Chicago, IL 60637, USA

Full list of author information is available at the end of the article
}

(c) The Author(s). 2020 Open Access This article is licensed under a Creative Commons Attribution 4.0 International License, which permits use, sharing, adaptation, distribution and reproduction in any medium or format, as long as you give appropriate credit to the original author(s) and the source, provide a link to the Creative Commons licence, and indicate if changes were made. The images or other third party material in this article are included in the article's Creative Commons licence, unless indicated otherwise in a credit line to the material. If material is not included in the article's Creative Commons licence and your intended use is not permitted by statutory regulation or exceeds the permitted use, you will need to obtain permission directly from the copyright holder. To view a copy of this licence, visit http://creativecommons.org/licenses/by/4.0/ The Creative Commons Public Domain Dedication waiver (http://creativecommons.org/publicdomain/zero/1.0/) applies to the data made available in this article, unless otherwise stated in a credit line to the data. 